\title{
LETTER
}

Przemysław Kościk • Jayanta K. Saha

\section{Ground-State Entanglement Properties of Helium Atom in a Finite Spherical Cavity}

Received: 20 March 2015 / Accepted: 3 May 2015 / Published online: 20 May 2015

(C) The Author(s) 2015. This article is published with open access at Springerlink.com

\begin{abstract}
The effects of the spatial confinement on the ground-state entanglement properties of the helium atom have been studied. The finite oscillator potential is used to represent the confining potential. The transition to the free atom regime and the harmonic confinement regime are discussed in detail in dependence of the control parameters of the system.
\end{abstract}

\section{Introduction}

Spatially confined systems of atoms have been a subject of intense interest since the birth of quantum mechanics. With the technological advancement, it is now possible to prepare control experiments with such systems in the laboratory. A detail discussion on these applications can be found in [1,2]. In particular, the research activity has expanded towards investigating properties of confined real two electron systems. The impact of confinement on the properties of helium atom and its iso-electronic ions was mainly addressed in literature in connection with impenetrable spherical walls [3-10]. Physical properties of such systems e.g. energy levels, ionization radius or correlation energy appear to be now well understood. Nonetheless, theoretical data for different confinement models are rather scarce. Some results for confinement models such as a harmonic potential or a Gaussian potential are available in Refs. [10-12] and [10,13], respectively.

In recent years, there has been an increase of interest in the study of quantum entanglement. Besides a pivotal role of the entanglement in the quantum information science and its related fields, it is gradually becoming an essential part of the study devoted to structural properties of few-body systems. In particular, it provides an alternative tool to quantify the quantum correlation between the indistinguishable constituent particles. Being the best candidate to study the behavior of quantum correlation between the fermions, the helium-like atoms have been in recent years intensively studied in contexts of their entanglement properties [14-21]. However, studies on entanglement properties of confined helium-like atoms are still limited.

The Hamiltonian for atomic systems with two electrons inside a cavity is given by

$$
H=\sum_{i=1}^{2}\left[-\frac{1}{2} \nabla_{i}^{2}+V\left(r_{i}\right)-\frac{Z}{r_{i}}\right]+\frac{1}{r_{12}},
$$

P. Kościk (ख)

Institute of Physics, Jan Kochanowski University, ul. Świętokrzyska 15, 25-406 Kielce, Poland

E-mail: koscik@pu.kielce.pl

J. K. Saha

Indian Association for the Cultivation of Science, Jadavpur, Kolkata 700032, India 
where $V(r)$ is an external potential. In this paper, we consider two models of confined helium atom $(Z=2)$, namely one model with a cavity modeled by a two-parameter family of weakly confining potential of the form

$$
V(r)=A-A\left(1+\frac{B}{\sqrt{A}} r\right) e^{-\frac{B}{\sqrt{A}} r},
$$

$(A>0, B \geq 0)$, and another one with a harmonic well

$$
V(r)=\frac{B^{2}}{2} r^{2},
$$

being a Taylor series of (2) around $r=0$ limited to second order term. For example, an attractive version of the potential (2), i.e. $V(r)-A$, has been recently used to model a quantum dot [22], to explore the effect of the confinement on the autoionizing resonance states of helium atom [23], and to study a photoionization cross sections of atomic impurities in spherical quantum dots [24].

The main purpose of the present paper is to make a detailed investigation of singlet ground-state entanglement properties of helium atom in the cavity (2). The structure of this letter is as follows. In Sect. 2 we introduce the procedure to compute the entanglement in the singlet S-symmetry states. Section 3 concerns our results and a brief summary of our conclusions is given in Sect. 4.

\section{The von Neumann entropy}

The characterization of entanglement of a pure fermion state is achieved by a Slater decomposition of its total wavefunction. It is is non-entangled if, and only if, it can be expressed by the one single Slater determinant [25]. Deviations from such a form is used to measure the amount of entanglement in the system. The wellknown entanglement measures are the von Neumann $(\mathrm{vN})$ entropy [26] or its approximation the so-called a linear entropy [27]. Here we are only interested in S-symmetry ground-state, the spatial wavefunction of which depends explicitly only on the radial coordinates $r_{1}, r_{2}$ and the inter-electronic angle coordinate $\theta$, $\psi\left(\mathbf{r}_{1}, \mathbf{r}_{2}\right) \equiv \psi\left(r_{1}, r_{2}, \cos \theta\right)$. The Schmidt decomposition of such a wavefunction can be written in a form [28-30]

$$
\psi\left(\mathbf{r}_{1}, \mathbf{r}_{2}\right)=\sum_{\substack{n=0 \\ l=0}}^{\infty} \sum_{m=-l}^{m=l} \frac{4 \pi k_{n l}}{2 l+1} u_{n l m}^{*}\left(\mathbf{r}_{1}\right) u_{n l m}\left(\mathbf{r}_{2}\right),
$$

where $u_{n l m}(\mathbf{r})=\frac{v_{n l}(r)}{r} Y_{l m}(\theta, \varphi)$ and $Y_{l m}$ are the spherical harmonics. The radial orbitals $v_{n l}(r)$ and the coefficients $k_{n l}$ are both real and can be determined by the integral equations [21]

$$
\int_{0}^{\infty} f_{l}\left(r, r^{\prime}\right) v_{n l}\left(r^{\prime}\right) d r^{\prime}=k_{n l} v_{n l}(r)
$$

with

$$
\begin{aligned}
f_{l}\left(r_{1}, r_{2}\right)= & r_{1} r_{2} \frac{2 l+1}{2} \\
& \times \int_{0}^{\pi} \psi\left(r_{1}, r_{2}, \cos \theta\right) P_{l}(\cos \theta) \sin \theta d \theta,
\end{aligned}
$$

where $P_{l}$ is the lth order Legendre polynomial. The Schmidt orbitals $u_{n l m}(\mathbf{r})$ are nothing but eigenvectors of the spatial reduced density matrix $(\mathrm{RDM})$

$$
\rho_{\text {red }}^{\text {spat }}\left(\mathbf{r}, \mathbf{r}^{\prime}\right)=\int\left[\psi\left(\mathbf{r}, \mathbf{r}_{1}\right)\right]^{*} \psi\left(\mathbf{r}^{\prime}, \mathbf{r}_{1}\right) d \mathbf{r}_{1},
$$

with eigenvalues (occupancies) $\lambda_{n l}$ related to the $k_{n l}$ coefficients in (4) by $\lambda_{n l}=\left(\frac{4 \pi k_{n l}}{2 l+1}\right)^{2}$, where due to the $2 l+1$ fold degeneracy, the normalization condition gives $\sum_{n l}(2 l+1) \lambda_{n l}=1$.

In this paper, we quantify the entanglement in terms of the $\mathrm{vN}$ entropy

$$
S_{v N}=S\left[\rho_{\text {red }}\right]-1,
$$


Table 1 Convergence of the ground-state energy for $A=5$ for different values of $B$ as a function of $N$

\begin{tabular}{llll}
\hline & $B=0.001$ & $B=1$ & $B=100$ \\
\hline$N=3$ & -2.9036963 & -2.2747300 & 7.0812853 \\
$N=5$ & -2.9037217 & -2.2747762 & 7.0812437 \\
$N=6$ & -2.9037227 & -2.2747767 & 7.0812417 \\
\hline
\end{tabular}

where $S\left[\rho_{\text {red }}\right]=-\operatorname{Tr}\left[\rho_{\text {red }} \log _{2} \rho_{\text {red }}\right]$ is the ordinary vN entropy. The vN entropy (7) vanishes when the corresponding total two-electron wavefunction is expressed as one single determinant [21]. Taking into account the degeneracies appearing in the spectrum of the RDM, the vN entropy of the singlet S state takes, in terms of the occupancies, the form [21]

$$
S_{v N}=-\sum_{n l}^{\infty}(2 l+1) \lambda_{n l} \log _{2} \lambda_{n l}
$$

One of most efficient ways to determine the coefficients $k_{n l}$ is to solve (5) through a discretization technique. Thus diagonalization of the matrix $\left[\Delta r f_{l}(i \Delta r, j \Delta r)\right]_{K \times K}, i, j=0,1, \ldots, K-1, \Delta r=R /(K-1)$, gives approximation to the $K$ lowest coefficients $k_{n l}$. For more details on this point, see [30].

\section{Numerical Results}

In order to compute the ground state energies and wave functions of (1) with (2) and with (3), we use here a Hylleraas variational wave function

$$
\psi_{H y l}=\sum_{n m p} c_{n m p}|n m p\rangle
$$

with $0 \leq n+m+p \leq N$, where $|n m p\rangle=e^{-\mu s} s^{n} t^{2 m} u^{p}, s=r_{1}+r_{2}, t=r_{2}-r_{1}, u=r_{12}$ and $\mu$ is a non-linear variational parameter.

In our calculations, the ground state energy $E$ and the corresponding coefficients $\mathbf{C}=\left[c_{n m p}\right]$ are determined by solving the generalized eigenvalue problem

$$
(\mathbf{H}-E \mathbf{S}) \mathbf{C}=0,
$$

where $\mathbf{S}=\left[\left\langle n^{\prime} m^{\prime} p^{\prime} \mid n m p\right\rangle\right]$ and $\mathbf{H}=\left[\left\langle n^{\prime} m^{\prime} p^{\prime}|H| n m p\right\rangle\right]$. Here the non-linear parameter $\mu$ is iteratively determined so as to minimize the approximate energy $\left(\partial E^{N} / \partial \mu=0\right)$.

We concluded from our calculations that a rate of convergence of the method only slightly depends on parameter values in (2). For the sake of demonstration, the ground state energies obtained as described above are shown in Table 1 for $A=5$ and for some different values of $B$ as a function of $N$. It is worth also mentioning here that the convergence is comparable to that in the case of the free helium atom, where its ground state energy value is recovered with six digits accuracy already at $N=6$ (84-terms), -2.9037233 .

Figures 1 and 2 show the results computed with 84 -terms $(N=6)$ wavefunctions for the ground state energy and the corresponding vN entropy, for different values of $A$ as a function of $B^{-1}$, where to highlight the changes with $B^{-1}$ a logarithmic coordinate has been used along abscissa. Each result presented in Fig. 2 , has been obtained with the sum (8) truncated up to $l=5$ and $n=176\left(\Delta r=32^{-1}, R=5.5\right)$, which was sufficient to get a good estimate of the true value of the vN entropy.

In the limit as $B \rightarrow \infty\left(\ln \left(B^{-1}\right) \rightarrow-\infty\right)$, the energy saturates at a constant value, being larger at larger $A$. This is attributed to the fact that in this limit the confining potential (2) tends to $V(r)=A$ and, as a consequence, the energy of the system is at the time equals the sum of the energy of the free helium atom plus $2 A$. When $B$ decreases $\left(\ln \left(B^{-1}\right)\right.$ increases $)$, the energy decreases until it saturates irrespectively of the value of $A$ at a free-helium energy value as $B \rightarrow 0\left(\ln \left(B^{-1}\right) \rightarrow \infty\right)$ that follows from the fact that in this limit $V(r)=0$. The fact that for a fixed value of $A$, both in the $B \rightarrow 0$ and $B \rightarrow \infty$ limit, the system behaves as a free helium atom is also reflected in the value of the vN entropy that attains, in both limits, a value of the ground state of the free helium atom, $S_{v N} \approx 0.0849$ [18,19]. Interestingly enough, one sees from both figures that the value of $B$ at which the system attains a free atom regime (corresponding to small $B$ values) only slightly depends on $A$. As a matter of fact, this occurs at $B \approx 0.135\left(\ln B^{-1} \approx 2\right)$, regardless of values of $A$. On the 


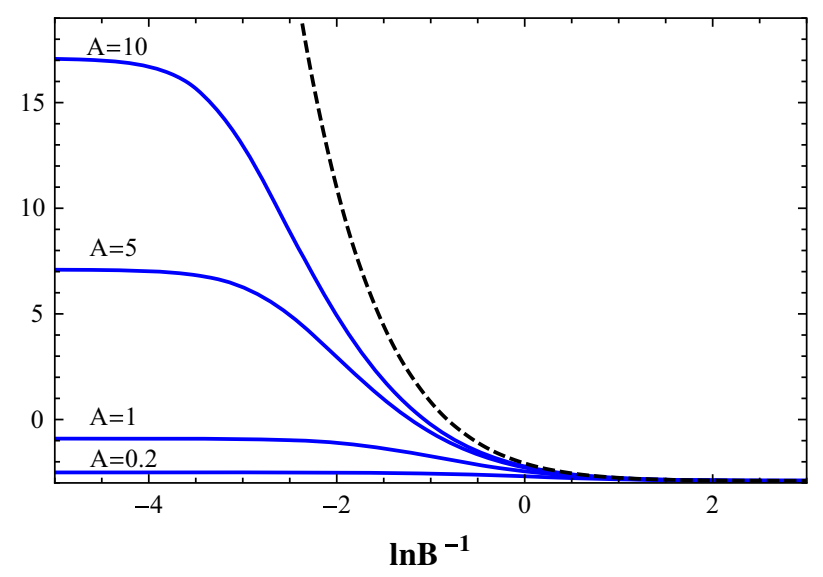

Fig. 1 The ground state energy as a function of $\ln B^{-1}$ for different values of $A$. The dashed line represents the results for the helium atom in the trap (3)

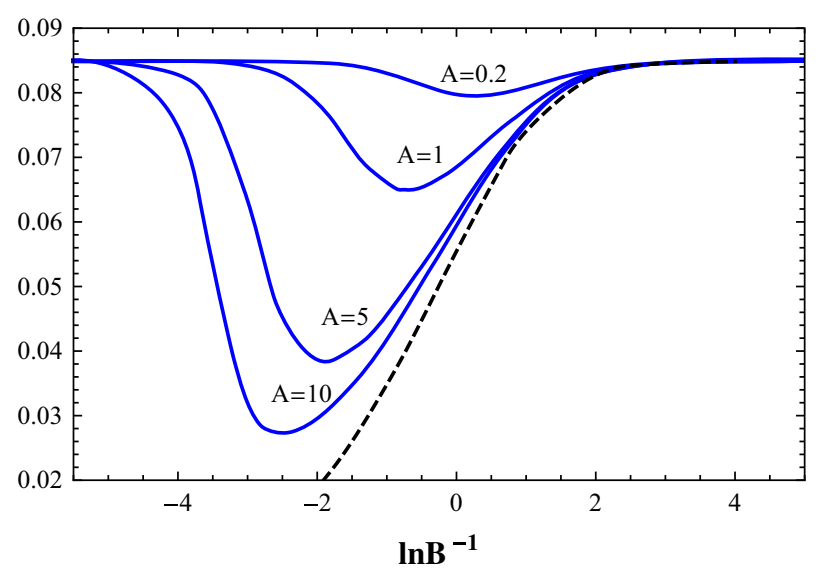

Fig. 2 The von Neumann entropy of the ground-state as a function of $\ln B^{-1}$ for different values of $A$. The dashed line represents the results for the helium atom in the trap (3)

other hand, the larger is the value of $A$, the larger is the $B$ value at which the free atom regime (corresponding to large $B$ values) is reached. As can be further inferred, the value of $B$ below which the helium atom under the cavity (2) behaves as the helium atom under the harmonic cavity (3) increases with the increase in $A$. This fact in turn can be understood by referring to the Taylor series expansion of the potential (2) around $r=0$, i.e.,

$$
V(r)=A \sum_{n=2}^{\infty}(-1)^{n}\left(\frac{n-1}{n !}\right)\left(\frac{B}{\sqrt{A}}\right)^{n} r^{n},
$$

(converged elsewhere), namely it follows from its form that the smaller is the value of $B / \sqrt{A}$, the larger is the range of $r$ in which (2) exhibits the harmonic behaviour (3).

\section{Conclusions}

We have investigated the impact of spatial confinement in the form of a two parameter finite spherical cavity on the ground state entanglement properties of Helium atom using trial variational wave function expanded in explicitly correlated Hylleraas basis. Our results showed the dependencies of the vN entropy on the control parameters of the system. In general, it turned out that the invasion of the spherical cavity decreases the entanglement. Among other things, it was found that the deeper is the cavity (larger $A$ ), the wider is the range of $B$ in which the von Neumann entropy makes its most rapid variations. 
Open Access This article is distributed under the terms of the Creative Commons Attribution 4.0 International License (http:// creativecommons.org/licenses/by/4.0/), which permits unrestricted use, distribution, and reproduction in any medium, provided you give appropriate credit to the original author(s) and the source, provide a link to the Creative Commons license, and indicate if changes were made.

\section{References}

1. Jaskólski, W.: Confined many-electron systems. Phys. Rep. 271, 1 (1996)

2. Sil, A.N., Canuto, S., Mukherjee, P.K.: Spectroscopy of confined atomic systems: effect of plasma. Adv. Quantum Chem. 58, 115 (2009)

3. Aquino, N., Flores-Riveros, A., Rivas-Silva, J.F.: The compressed helium atom variationally treated via a correlated Hylleraas wave function. Phys. Lett. A 307, 326 (2003)

4. Montgomery, H.E. Jr., Aquino, N., Flores-Riveros, A.: The ground state energy of a helium atom under strong confinement. Phys. Lett. A 374, 2044 (2010)

5. Flores-Riveros, A., Aquino, N., Montgomery, H.E. Jr.: Spherically compressed helium atom described by perturbative and variational methods. Phys. Lett. A 374, 1246 (2010)

6. Flores-Riveros, A., Rodriguez-Contreras, A.: Compression effects in helium-like atoms $(\mathrm{Z}=1, \ldots, 5)$ constrained by hard spherical walls. Phys. Lett. A 372, 6175 (2008)

7. Wilson, C.L., Montgomery, H.E. Jr., Sen, K.D., Thompson, D.C.: Electron correlation energy in confined two-electron systems. Phys. Lett. A 374, 4415 (2010)

8. Bhattacharyya, S., Saha, J.K., Mukherjee, P.K., Mukherjee, T.K.: Precise estimation of the energy levels of two-electron atoms under spherical confinement. Phys. Scr. 87, 065305 (2013)

9. Montgomery, H.E. Jr., Pupyshev, V.I.: Confined helium: excited singlet and triplet states. Phys. Lett. A 377, 2880 (2013)

10. Laughlin, C., Chu, S.I.: A highly accurate study of a helium atom under pressure. J. Phys. A Math. Theor. 42, 265004 (2009)

11. Sako, T., Diercksen, G.H.F.: Confined quantum systems: spectral properties of the atoms helium and lithium in a power series potential. J. Phys. B At. Mol. Opt. Phys. 36, 1433 (2003)

12. Sako, T., Diercksen, G.H.F.: Confined quantum systems: a comparison of the spectral properties of the two-electron quantum dot, the negative hydrogen ion and the helium atom. J. Phys. B: At. Mol. Opt. Phys. 36, 1681 (2003)

13. Wen- Fang, X.: A helium atom confined by a spherical Gaussian potential well. Commun. Theor. Phys. 49, 1287 (2008)

14. Dehesa, J.S. et al.: Quantum entanglement in helium. J. Phys. B At. Mol. Opt. Phys. 45, 015504 (2012)

15. Benenti, G., Siccardi, S., Strini, G.: Entanglement in helium. Eur. Phys. J. D 67, 83 (2013)

16. Lin, Y., Lin, C., Ho, Y.K.: Spatial entanglement in two-electron atomic systems. Phys. Rev. A 87, 022316 (2013)

17. Lin, C.H., Lin, Y.C., Ho, Y.K.: Quantification of linear entropy for quantum entanglement in He, H- and Ps- ions using highly-correlated Hylleraas functions. Few-Body Syst. 54, 2147 (2013)

18. Kościk, P., Okopińska, A.: Entanglement entropies in the ground states of helium-like atoms. Few-Body Syst. 55, 1151 (2014)

19. Lin, C.H., Ho, Y.K.: Quantification of entanglement entropy in helium by the Schmidt-Slater decomposition method. FewBody Syst. 55, 1141 (2014)

20. Lin, C.H., Ho, Y.K.: Calculation of von Neumann entropy for hydrogen and positronium negative ions. Phys. Lett. A 378, 2861 (2014)

21. Kościk, P.: Entanglement in S states of two-electron quantum dots with Coulomb impurities at the center. Phys, Lett. A 377, 2393 (2013)

22. Kimani, P., Jones, P., Winkler, P.: Correlation studies in weakly confining quantum dot potent. Int. J. Quantum Chem. 108, 2763 (2008)

23. Chakraborty, S., Ho, Y.K.: Autoionization resonance states of two-electron atomic systems with finite spherical confinement. Phys. Rev A 84, 032515 (2011)

24. Lin, C.Y., Ho, Y.K.: Photoionization cross sections of hydrogen impurities in spherical quantum dots using the finite-element discrete-variable representation. Phys. Rev A 84, 023407 (2011)

25. Ghirardi, G., Marinatto, L.: General criterion for the entanglement of two indistinguishable particles. Phys. Rev. A 70, 012109 (2004)

26. Paškauskas, R., You, L.: Quantum correlations in two-boson wave functions. Phys. Rev. A 64, 042310 (2001)

27. Buscemi, F., Bordone, P., Bertoni, A.: Linear entropy as an entanglement measure in two-fermion systems. Phys. Rev. A 75, $032301(2007)$

28. Davidson, E.R.: Natural expansions of exact wavefunctions. III. The helium-atom ground state. J. Chem. Phys. 39, 875 (1964)

29. Davidson, E.R.: Properties and uses of natural orbitals. Rev. Mod. Phys. 44, 451 (1972)

30. Wang, J., Law, C.K., Chu, M.C.: S-wave quantum entanglement in a harmonic trap. Phys. Rev. A 72, 022346 (2005) 